06

\title{
Поверхностные и объемные волны в структуре ферродиэлектрик-магнитоактивный метаматериал
}

\author{
(ㄱ Л.Д. Филатов, Д.И. Семенцов \\ Ульяновский государственный университет, \\ 432970 Ульяновск, Россия \\ ฯ e-mail: sementsovdi@mail.ru
}

(Поступило в Редакцию 11 ноября 2015 г. В окончательной редакции 27 июня 2016 г.)

Исследована возможность распространения поверхностных волн на границе ферродиэлектрика и метаматериала, состоящего из решетки аморфных ферромагнитных микропроводков, помещенных в диэлектрическую матрицу. Получены и проанализированы дисперсионные зависимости для управляемой внешним магнитным полем ТЕ волны, найдены частотные области, в которых реализованы поверхностные, т. е. локализованные, частично локализованные и объемные волны. Для поверхностных волн определены основные волновые характеристики. Показано также, что ТМ волна в рассматриваемой структуре поверхностной быть не может.

DOI: 10.21883/JTF.2017.01.44020.1655

\section{Введение}

В последнее время большое внимание уделяется исследованию электро-динамических свойств метаматериалов и различных волновых процессов в них [1-6]. К таким волновым процессам относятся поверхностные волны, локализованные вблизи границы раздела двух сред, одной из которых является метаматериал [7-10]. Известно, что в области частот, где диэлектрическая или магнитная проницаемости (ДП и МП) одной из граничащих сред принимает отрицательные значения, вдоль плоской границы раздела возможно распространение поверхностных волн или поверхностных поляритонов (ПП) [11,12]. В немагнитных структурах за счет отрицательности ДП одной из сред реализуются ПП с поляризацией ТМ типа, которые не управляются магнитным полем [13-15]. Для реализации в структуре ПП, управляемых магнитным полем, МП одной из сред в рабочем частотном диапазоне должна принимать отрицательные значения [16-18].

Важное прикладное значение приобретают метаматериалы, реализуемые на основе решеток из микропроводов в непроводящей матрице [19-22]. Так, в работе [21] реализована структура на основе матрицы из стекла и микропроводков из ферромагнитного аморфного сплава $\mathrm{FeCoCrBSi}$, ее модель использовалась в работе [5]. В настоящей работе обсуждаются условия существования и волновые характеристики объемных и поверхностных волн в структуре с плоской границей раздела подобного метаматериала и ферродиэлектрика. Материальные параметры обоих сред в исследуемом частотном диапазоне зависят от приложенного магнитного поля. Под ферродиэлектриком мы понимаем материал, обладающий спонтанным магнитным моментом и не обладающий проводимостью, т.е. поглощением [23,24]. В СВЧ диапазоне данному определению наиболее полно отвечает феррит $\mathrm{Y}_{3} \mathrm{Fe}_{5} \mathrm{O}_{12}$, параметры которого далее мы будем использовать при численном анализе.

\section{1. Материальные соотношения}

Будем считать, что область $z<0$ занимает феррит, для которого ДП $\varepsilon_{f}$ в исследуемом СВЧ диапазоне является константой. Область $z>0$ занимает метаматериал, представляющий собой помещенную в диэлектрическую матрицу с ДП $\varepsilon_{d}$ решеточную структуру из параллельных оси $X$ магнитных микропроводков радиуса $a$, расположенных на расстоянии $b$ друг от друга. При статическом магнитном поле $H$, параллельном оси $Y$, тензоры МП каждой из сред имеют отличные от нуля диагональные компоненты $\mu_{x x}=\mu_{z z}=\mu_{\alpha}, \mu_{y y}=1$ и недиагональные $\mu_{x z}=-\mu_{z x}=i k_{\alpha}$ (индексы $\alpha=m, f$ относятся к метаматериалу и ферриту), где

$$
\mu_{m, f}=1+\frac{\omega_{m, f} \omega_{0}}{\omega_{0}^{2}-\omega^{2}}, \quad k_{m, f}=\frac{\omega \omega_{m, f}}{\omega_{0}^{2}-\omega^{2}} .
$$

Здесь введены характерные частоты структуры $\omega_{m}=$ $=(2 \pi a / b)^{2} \gamma M_{c}, \omega_{f}=4 \pi \gamma M_{f}$ и частота магнитного резонанса $\omega_{0}=\gamma H$, где $\gamma-$ магнитомеханическое отношение, $M_{f}$ и $M_{c}$ - намагниченности феррита и магнитных микропроводков.

В случае распространения волны вдоль оси $X$ константы распространения в каждой из сред $\beta_{\alpha}=k_{0} \sqrt{\varepsilon_{\alpha} \mu_{\alpha}^{\perp}}$. В случае поперечного (по отношению к магнитному полю) распространения волны эффективная магнитная проницаемость феррита имеет вид [24]

$$
\mu_{f}^{\perp}=1+\frac{\omega_{f}\left(\omega_{0}+\omega_{f}\right)}{\omega_{0}\left(\omega_{0}+\omega_{f}\right)-\omega^{2}} .
$$

Если длина распространяющейся в структуре волны $\lambda \gg b$, метаматериал можно считать однородной средой со средней намагниченностью $M_{m}=\eta M_{e}$, где $M_{e}-$ намагниченность материала проводков, а $\eta=\pi a^{2} / b^{2}-$ объемная концентрация проводника в среде. В случае поперечного подмагничивания эффективную МП метаматериала можно определить выражением [5]

$$
\mu_{m}^{\perp}=\left(1+\frac{\omega_{m}\left(\omega_{0}+\omega_{m}\right)}{\omega_{0}\left(\omega_{0}+\omega_{m}\right)-\omega^{2}}\right) F(\omega, \sigma) .
$$


Зависимость величины $\mu_{m}^{\perp}$ от проводимости проводков $\sigma$ определяется функцией

$$
F(\omega, \sigma)=\frac{1}{2}\left(1+\sqrt{1+\left(\frac{4 \pi \eta \sigma}{\varepsilon_{d} \omega}\right)^{2}}\right) .
$$

С точки зрения симметрии, при заданной ориентации проводков метаматериал может быть отнесен к одноосным кристаллам с диагональным тензором ДП с компонентами, вид которых зависит от типа волны, распространяющейся в среде. Так, составляющей волнового поля $E_{x}$ отвечает компонента тензора $\varepsilon_{x}=\varepsilon_{\|}$, a составляющим $E_{y}$ и $E_{z}$ отвечают компоненты $\varepsilon_{y}=\varepsilon_{z}=\varepsilon_{\perp}$. Указанные компоненты представляются в виде

$$
\begin{gathered}
\varepsilon_{\|}=(1-\eta) \varepsilon_{d}+\eta \varepsilon_{c} \approx \varepsilon_{d}+\eta \varepsilon_{c}, \\
\varepsilon_{\perp}=\frac{\varepsilon_{d} \varepsilon_{c}}{(1-\eta) \varepsilon_{c}+\eta \varepsilon_{d}} \approx \varepsilon_{d},
\end{gathered}
$$

где $\varepsilon_{c}-$ ДП проводника, которая в пренебрежении потерями запишется в виде

$$
\varepsilon_{c}=\varepsilon_{0}-\left(\omega_{p} / \omega\right)^{2},
$$

где $\varepsilon_{0}$ - вклад кристаллической решетки проводника, а плазменная частота для микропроводков определяется соотношением $[7,19]$

$$
\omega_{p}^{2}=\frac{2 \pi c^{2}}{b^{2} \ln (b / 2 \pi a)} .
$$

Приближенные соотношения (5) записаны с учетом малости величины $\eta$.

На рис. 1 приведены частотные зависимости эффективной МП метаматериала $\mu_{m}^{\perp}$ и феррита $\mu_{f}^{\perp}$ (сплошная и штриховая линии), а также частотная зависимость компонент ДП метаматериала $\varepsilon_{\|}$и $\varepsilon_{\perp}$ (сплошная и штриховая линии). При построении указанных зависимостей использовались следующие параметры: для метаматериала $M_{c}=500 \mathrm{Gs}, \sigma=10^{16} \mathrm{~s}^{-1}, \varepsilon_{d}=2.25$, $\varepsilon_{0}=2.5, a=10^{-3} \mathrm{~cm}, b=10^{-1} \mathrm{~cm}, \omega_{p}=1.25 \cdot 10^{12} \mathrm{~s}^{-1}$, $\omega_{m}=3.47 \cdot 10^{7} \mathrm{~s}^{-1}$, для феррита намагниченность насыщения $4 \pi M_{f}=1760 \mathrm{Gs}, \gamma=1.76 \cdot 10^{7}(\mathrm{~s} \cdot \mathrm{Oe})^{-1} \varepsilon_{f}=14$ (в СВЧ диапазоне). На рисунке приведены несколько характерных частот, каждая из которых определяется следующим образом. Частота $\omega_{1}=\sqrt{\omega_{0}\left(\omega_{0}+\omega_{m}\right)}$ является резонансной частотой метаматериала и определяется полюсом функции $\mu_{m}^{\perp}$, а частота $\omega_{2}=\omega_{0}+\omega_{m}-$ нулем этой функции. Аналогичные частоты определяются и для поперечно намагниченного феррита: $\mu_{f}^{\perp} \rightarrow \infty$ имеет место при $\omega_{3}=\sqrt{\omega_{0}\left(\omega_{0}+\omega_{f}\right)}, \mu_{f}^{\perp}=0$ при $\omega_{4}=\omega_{0}+\omega_{f}$. На частоте

$$
\omega_{5}=\omega_{p} / \sqrt{\varepsilon_{0}+\left(\eta^{-1}-1\right) \varepsilon_{d}}
$$

величина $\varepsilon_{\|}$обращается в нуль, а при $\omega \leq \omega_{5}$ она становится отрицательной.

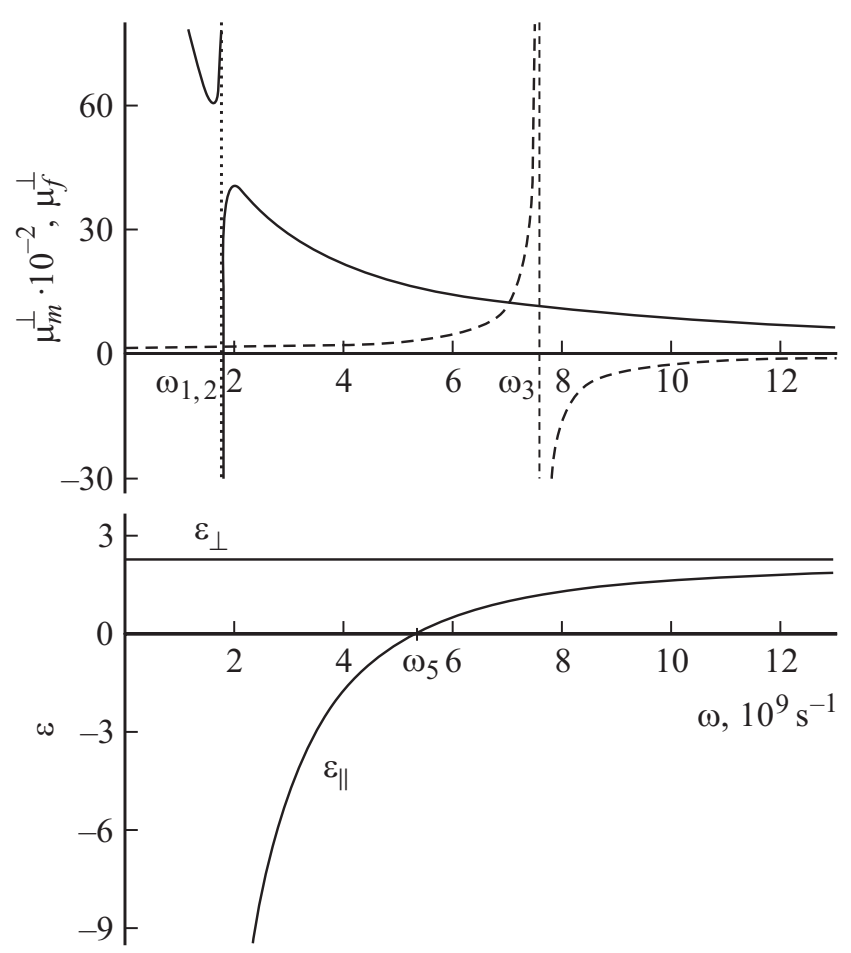

Рис. 1. Частотные зависимости МП $\mu_{m}^{\perp}$ и $\mu_{f}^{\perp}$ (сплошная и штриховая линии) и ДП $\varepsilon_{\perp}$ и $\varepsilon_{\|}$(сплошная и штриховая линии).

Эффективными параметрами метаматериала можно управлять с помощью внешнего магнитного поля, изменяя частоту $\omega_{0}$. Так, при фиксированной частоте $\mu_{m}^{\perp}$ принимает отрицательные значения в области значений поля

$$
\left(\omega-\omega_{m}\right) / \gamma<H<\left(\sqrt{\omega_{m}^{2}+4 \omega^{2}}-\omega_{m}\right) / 2 \gamma .
$$

Наличие частотных областей, для которых $\mu_{m}^{\perp}<0$, $\mu_{f}^{\perp}<0$ и $\varepsilon_{\|}<0$, указывает на возможность реализации в структуре поверхностных волн плазмон-поляритонного типа обеих ортогональных поляризаций ТМ и ТЕ.

\section{2. Дисперсионное соотношение для ТМ волны}

В этом случае волновыми компонентами поля являются $E_{x}, H_{y}, E_{z}$. Для указанной поляризации в волновые уравнения для каждой из граничащих сред входит компонента тензора МП $\mu_{y y}$, которая при выбранной ориентации внешнего магнитного поля от величины этого поля не зависит и равна единице. Поэтому на динамику волны этого типа внешнее поле не влияет. С учетом гармонических зависимостей волновых полей от времени их величины пропорциональны фактору $\exp (i \omega t)$, который далее опускаем. При этом уравнения для тангенциальных (по отношению к границе раздела 


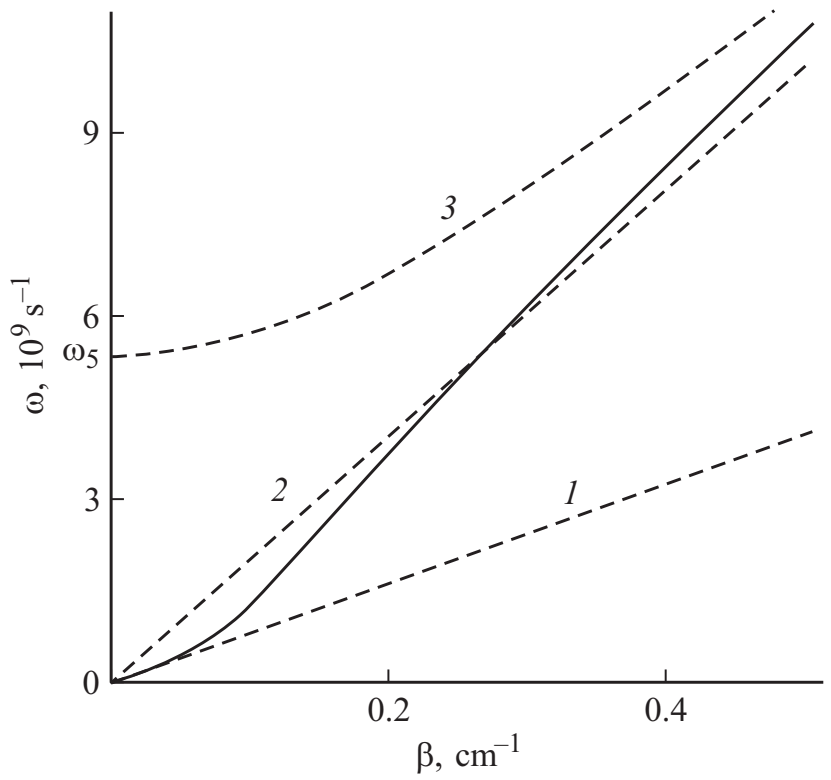

Рис. 2. Дисперсионная зависимость $\omega(\beta)$ для объемной ТМ волны в структуре.

сред) компонент волнового поля имеют вид

$$
\frac{d^{2} H_{y}}{d z^{2}}-q_{f, m}^{2} H_{y}=0, \quad E_{x}=-\frac{i}{k_{0} \varepsilon_{f, m}} \frac{d H_{y}}{d z},
$$

где поперечные компоненты волнового вектора в каждой из сред

$$
q_{f}^{2}=\beta^{2}-k_{0}^{2} \varepsilon_{f}, \quad q_{m}^{2}=\left(\varepsilon_{\|} / \varepsilon_{\perp}\right)\left(\beta^{2}-k_{0}^{2} \varepsilon_{\perp}\right),
$$

где $\beta$ - продольная компонента волнового вектора (константа распространения), $k_{0}=\omega / c, c-$ скорость света в вакууме, $\varepsilon_{m}=\varepsilon_{x}=\varepsilon_{\|}$. Если волна является поверхностной, то ее магнитное поле может быть представлено в виде

$$
H_{y}(x, z)=H_{0} \exp (-i \beta x) \begin{cases}\exp \left(-q_{m} z\right), & z>0, \\ \exp \left(q_{f} z\right), & z<0 .\end{cases}
$$

С учетом (12) и граничного условия $E_{x 1}(0)=E_{x 2}(0)$ приходим к дисперсионным соотношениям для этого типа волны

$$
\frac{q_{f}}{\varepsilon_{f}}+\frac{q_{m}}{\varepsilon_{\|}}=0, \quad \beta=k_{0} N_{\mathrm{eff}}=k_{0} \sqrt{\frac{\varepsilon_{f} \varepsilon_{\perp}\left(\varepsilon_{f}-\varepsilon_{\|}\right)}{\varepsilon_{f}^{2}-\varepsilon_{\perp} \varepsilon_{\|}}},
$$

где $N_{\text {eff }}$ - эффективный показатель преломления ТМ волны в рассматриваемой стуктуре. Для волны, локализованной вблизи границы раздела со стороны обеих сред, должны выполняться условия $q_{m, f}>0$. Так как $\varepsilon_{f}>0$, в частотной области существования ПП необходимо выполнение условия $\varepsilon_{\|}<0$. Такая область существует $\left(\omega<\omega_{5}\right)$, однако для этой области в соответствии с (11) $q_{m}^{2}<0$, что противоречит условию существования поверхностной волны.
Дисперсионные соотношения (13) описывают поведение волны ТМ типа, которая может быть локализованной вблизи границы раздела только в феррите, тогда как в метаматериале она остается объемной. Зависимость $\omega(\beta)$ для волны этого типа представлена на рис. 2 сплошной кривой. Асимптотами для этой кривой в разных областях частотного спектра являются две линейные зависимости $\omega=c_{d, f} \beta$ (штриховые линии 1 и 2) - фотонные линии в матрице метаматериала и в феррите, а также зависимость $\omega=c_{\|} \beta$ (штриховая кривая 3) - дисперсионная кривая для объемной волны в эффективной среде с ДП $\varepsilon_{\|}(\omega)$. Здесь $c_{d, f}=c / \sqrt{\varepsilon_{d, f}}$ и $c_{\|}(\omega)=c / \sqrt{\varepsilon_{\|}(\omega)}-$ фазовые скорости объемных волн в соответствующих средах.

\section{3. Дисперсионное соотношение для TE волны}

В этом случае волновыми компонентами поля являются $H_{x}, E_{y}, H_{z}$. При этом уравнения для тангенциальных компонент волнового поля имеют вид

$$
\frac{d^{2} E_{y}}{d z^{2}}-q_{f, m}^{2} E_{y}=0, \quad H_{x}=-\frac{i}{k_{0} \mu_{\perp}^{\alpha}}\left(\frac{d E_{y}}{d z}+\frac{\kappa_{\alpha}}{\mu_{\alpha}} \beta E_{y}\right),
$$

где поперечные компоненты волнового вектора в каждой из сред

$$
q_{f}^{2}=\beta^{2}-k_{0}^{2} \varepsilon_{f} \mu_{\perp}^{f}, \quad q_{m}^{2}=\beta^{2}-k_{0}^{2} \varepsilon_{\perp} \mu_{\perp}^{m} .
$$

Координатную зависимость электрического поля этой поверхностной волны запишем в виде

$$
E_{y}(x, z)=E_{m} \exp (-i \beta x) \begin{cases}\exp \left(-q_{m} z\right), & z>0 \\ \exp \left(q_{f} z\right), & z<0\end{cases}
$$

где $k_{0}=\omega / c, c-$ скорость света в вакууме. Учитывая равенство $H_{x 1}(0)=H_{x 2}(0)$, приходим к дисперсионному соотношению

$$
\frac{q_{m}}{\mu_{m}^{\perp}}+\frac{q_{f}}{\mu_{f}^{\perp}}=\beta G(\omega, H)
$$

где зависящая от частоты и внешнего поля функция $G(\omega, H)$ имеет вид

$$
G(\omega, H)=\frac{\kappa_{m}}{\mu_{m} \mu_{m}^{\perp}}-\frac{\kappa_{f}}{\mu_{f} \mu_{f}^{\perp}} .
$$

Уравнение (17) можно представить следующим образом:

$$
A \beta^{4}+B \beta^{2}-C=0,
$$

где введены обозначения:

$$
\begin{aligned}
A= & {\left[\left(\kappa_{m} / \mu_{m} \mu_{m}^{\perp}-\kappa_{f} / \mu_{f} \mu_{f}^{\perp}\right)^{2}-\left(\mu_{m}^{\perp}\right)^{-2}-\left(\mu_{f}^{\perp}\right)^{-2}\right]^{2} } \\
& -4 /\left(\mu_{m}^{\perp} \mu_{f}^{\perp}\right)^{2},
\end{aligned}
$$




$$
\begin{gathered}
B=4\left(\mu_{f}^{\perp} \varepsilon_{f}+\mu_{m}^{\perp} \varepsilon_{\perp}\right) /\left(\mu_{m}^{\perp} \mu_{f}^{\perp}\right)^{2}+2\left(\varepsilon_{\perp} / \mu_{m}^{\perp}+\varepsilon_{f} / \mu_{f}^{\perp}\right) \\
\times\left[\left(\kappa_{m} / \mu_{m} \mu_{m}^{\perp}-\kappa_{f} / \mu_{f} \mu_{f}^{\perp}\right)^{2}-\left(\mu_{m}^{\perp}\right)^{-2}-\left(\mu_{f}^{\perp}\right)^{-2}\right], \\
C=\left(\varepsilon_{\perp} / \mu_{m}^{\perp}-\varepsilon_{f} / \mu_{f}^{\perp}\right)^{2} .
\end{gathered}
$$

Решение уравнения (19) можно представить в виде

$$
\beta_{ \pm}^{2}=k_{0}^{2} \frac{-B \pm \sqrt{B^{2}+4 A C}}{2 A} .
$$

Анализ приведенных выражений показывает, что распространяющемуся ПП может отвечать только одна ветвь $\beta_{+}$, для которой на всем интервале частот $\beta_{+}^{2}>0$ (далее $\beta_{+}$будем обозначать $\beta$ ).

Одной из важных характеристик является глубина залегания поля поверхностной волны в каждой из сред, которая в отсутствие поглощения определяется выражением $\lambda=1 / q$. На характер волны в структуре указывает знак величины $q^{2}$ в каждой из сред. Там, где $q^{2}$ положительно, и для феррита, и для метаматериала реализуется поверхностная волна. Видно, что в спектре имеется только один узкий частотный интервал $\omega_{1}<\omega<\omega_{2}$, где это условие выполняется. Вне этого интервала реализуются состояния либо с частичной локализацией волны со стороны одной из сред (это области $\left(\omega_{2}, \omega_{f}\right)$ и $\left.\omega>\omega_{4}\right)$, либо с отсутствием локализации, когда в структуре распространяется объемная волна (это область $\omega_{f}<\omega<\omega_{4}$ ). Видно, что в узкой частотной области $\omega_{1}<\omega<\omega_{2}$ наблюдается максимум локализации и следующий за ним резкий переход волны из поверхностной в объемную. Степенью локализации поля ТЕ волны можно управлять с помощью внешнего магнитного поля, которое реализует перестройку дисперсионного спектра ПП.

\section{4. Численный анализ}

В отличие от стандартного дисперсионного соотношения (13) для поверхностных волн с нулевой правой частью, которое требует отрицательности диэлектрической проницаемости одной из сред, в случае волны ТЕ типа знак правой части уравнения (17) может быть как отрицательным, так и положительным. Знак величины $G$ меняется с ростом частоты, и выше частоты $\omega_{2}$ эта величина остается положительной. С учетом возможности разного знака величины $G(\omega, H)$ требованием существования ПП в структуре является только положительность величин $q_{m}^{2}$ и $q_{f}^{2}$, частотная зависимость которых приведена на рис. 3 (сплошная и штриховая кривые). Существование в структуре ПП возможно в частотных областях, отвечающих совместному выполнению условий $q_{m}^{2}>0$ и $q_{f}^{2}>0$. В соответствии с приведенными зависимостями такие области могут находиться лишь в узком частотном интервале, выделенном на рисунке рамкой. На остальных частотных интервалах распространяющаяся в структуре волна является либо полностью объемной (где $q_{m}^{2}<0$ и $q_{f}^{2}<0$ ), либо локализованной вблизи границы раздела со стороны феррита (где $q_{m}^{2}<0$, а $q_{f}^{2}>0$ ).
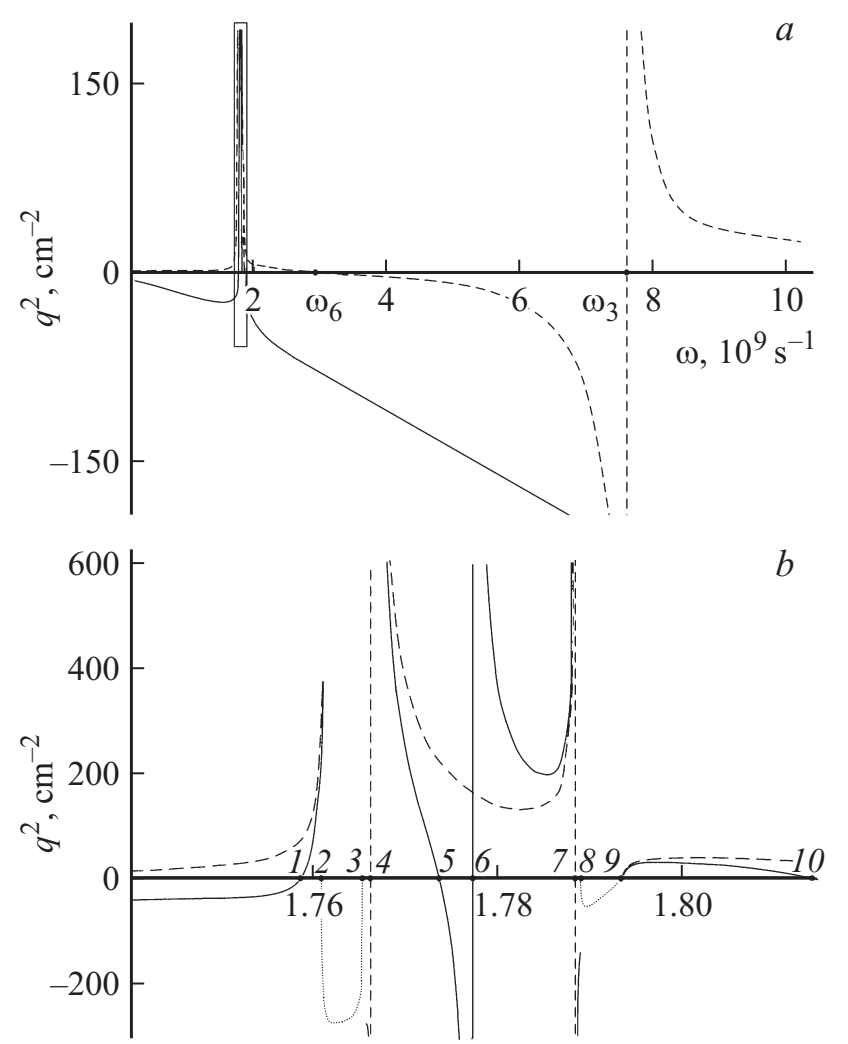

Рис. 3. Частотные зависимости величин $q^{2}$ для метаматериала и феррита (сплошная и штриховая линии) на большом $(a)$ и малом $(b)$ частотном интервалах, пунктирная линия $\operatorname{Im} q^{2}(\omega)$ на интервале $\left(\omega_{1}, \omega_{2}\right)$.

В увеличенном масштабе приведены частотные зависимости величин $q_{m}^{2}$ и $q_{f}^{2}$ на частотном интервале, выделенном на рис. $3, a$ рамкой. Видно, что ПП могут распространяться в структуре на частотных участках $(1,2),(4,5),(6,7)$ и $(9,10)$. На интервалах $(2,3)$ и $(4,5)$ величины $q_{m}^{2}$ и $q_{f}^{2}$ становятся равными и чисто мнимыми, при этом $q_{m}=q_{f}=(1-i) q / \sqrt{2}$, где $q-$ действительная величина. Это означает, что распространяющаяся вдоль границы раздела сред волна в указанных частотных интервалах также является поверхностной с экспоненциально спадающей амплитудой и зависящей от координаты $z$ фазой.

На рис. 4 приведены дисперсионные зависимости $\omega(\beta)$ для ТЕ волны, полученные при значениях внешнего магнитного поля $H_{0}=(100,115)$ Ое (сплошные и штриховые кривые). Окрестность области аномальной дисперсии $\omega_{1}<\omega<\omega_{2}$, в которой проницаемость метаматериала $\mu_{m}^{\perp}<0$ приведена в увеличенном по частоте масштабе. Из приведенных зависимостей следует, что с ростом внешнего поля указанная область смещается в область более высоких частот. Как было показано выше, существование ПП возможно лишь в области $\omega_{1}<\omega<\omega_{2}$. На остальных участках спектра имеет место либо частичная локализация волнового поля вблизи границы раздела со стороны одной из сред, либо реализуется объемная волна. 

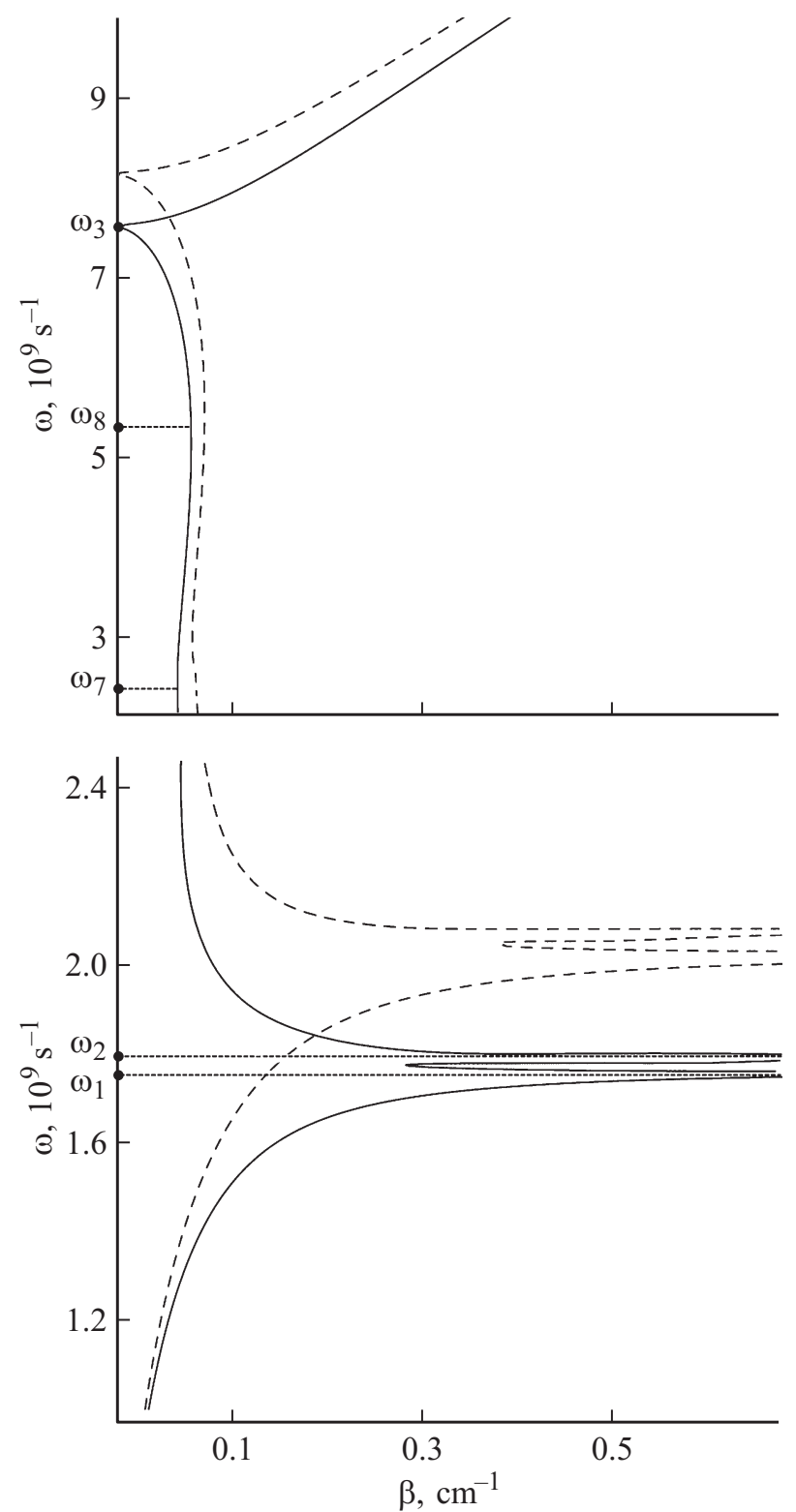

Рис. 4. Дисперсионные зависимости $\omega(\beta)$ для ТЕ волны при $H=(100,150)$ Ое (сплошная и штриховая кривые).

Рассматриваемый тип волны является магнитоуправляемым. На рис. 5 представлена зависимость от внешнего магнитного поля константы распространения, которая отвечает частотам $\omega=(1.77,2.1) \cdot 10^{9} \mathrm{~s}^{-1}$ (рис. 5, $a$, кривые 1,2$)$. На рис. $5, b$ в увеличенном по полю масштабе приведен участок указанной зависимости (для $\left.\omega=2.1 \cdot 10^{9} \mathrm{~s}^{-1}\right)$, на котором точками выделены характерные поля $h_{i}$. Ниже приведены выражения для этих полей

$h_{1,3}=$

$$
\begin{gathered}
\frac{\sqrt{4\left[\omega(1+F) \mp\left(F \omega_{m}-\omega_{f}\right)\right](F+1) \omega-\left(F \omega_{m}+\omega_{f}\right)^{2}}-F \omega_{m}-\omega_{f}}{2 \gamma(F+1)}, \\
h_{2}=\frac{\omega}{\gamma}+\frac{\omega_{f}-F \omega_{m}}{\gamma(F-1)}, \quad h_{4}=\frac{\omega}{\gamma} .
\end{gathered}
$$

Указанные поля для частоты $\omega=2.1 \cdot 10^{9} \mathrm{~s}^{-1}$ принимают следующие значения: $h_{1-4}=117.35,117.77,118.9$, 119.32 Ое. Поля $h_{2-4}$ определяют асимптотические значения для дисперсионных ветвей $\beta(H)$ при фиксированной частоте.

Одной из важных характеристик является глубина залегания поля волны в каждой из сред, которая в отсутствие поглощения определяется выражением $\lambda_{m, f}=1 / q_{m, f}$. Если обе величины $q_{m, f}$ действительны, то глубина залегания поля волны в каждой из сред конечна и возможно существование поверхностной волны. Возможны также состояния с одной действительной величиной $-q_{f}$. В этом случае имеет место частичная локализация волны - только со стороны феррита. Если же $q_{m, f}$ являются чисто мнимыми, то локализация отсутствует и в структуре распространяется объемная волна. Максимумы локализации наблюдаются на частотах, близких к точкам 4 (справа) и 7 (слева). Минимальные значения глубины залегания $\lambda_{m} \approx 1.35 \cdot 10^{-2} \mathrm{~cm}$ и $\lambda_{f} \approx 1.51 \cdot 10^{-2} \mathrm{~cm}$. При переходе через эти точки наблюдается резкий переход поверхностной волны в объемную.

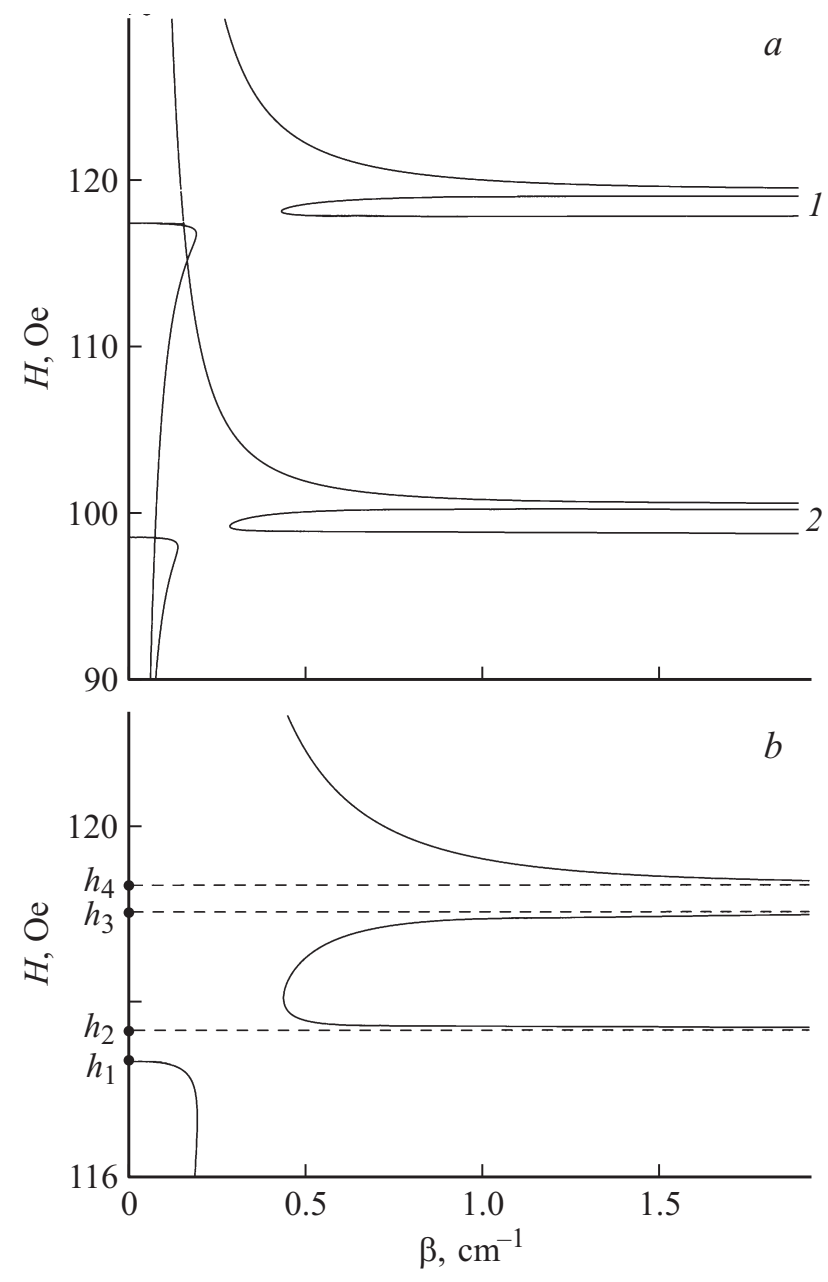

Рис. 5. Полевая зависимость константы распространения ТЕ волны при $\omega=(1.77,2.1) \cdot 10^{9} \mathrm{~s}^{-1}(a$, кривые 1,2$)$ и увеличенная по масштабу зависимость на частоте $\omega=2.1 \cdot 10^{9} \mathrm{~s}^{-1}(b)$. 


\section{Групповая и фазовая скорости}

На основе приведенных выше дисперсионных соотношений построены частотные зависимости фазовой $v_{p h}=\omega / \beta=c / N_{\text {eff }}$ и групповой скоростей собственных волн структуры. Для ТМ волны групповая скорость [25]

$$
v_{g}=\frac{d \omega}{d \beta}=c\left(N_{\mathrm{eff}}+\omega d N_{\mathrm{eff}} / d \omega\right)^{-1}
$$

на исследуемом участке спектра положительна и больше фазовой скорости, так как в рассматриваемой области частот для данного типа волны реализуется нормальная дисперсия, т.е. $d N_{\mathrm{eff}} / d \omega>0$. Из представленных на рис. 6 зависимостей следует, что на всем частотном интервале $v_{g}<v_{p h}$, но с ростом частоты обе скорости асимптотически стремятся к величине $v_{\mathrm{TM}}=c / \sqrt{\varepsilon_{d}+\eta \varepsilon_{0}}$, которая для выбранных параметров структуры принимает значение $v_{\mathrm{TM}}=0.67 \mathrm{c}$. При этом существенного замедления ТМ волна не испытывает.

Для ТЕ волны в исследуемом диапазоне имеется несколько частот, на которых наблюдаются особенности в поведении групповой и фазовой скоростей (рис. 7). Так, на частотах $\omega_{1}, \omega_{2}$ и $\omega_{3}$ имеет место стремление к нулю групповой скорости со сменой знака и фазовой - без смены знака, что указывает на существенное замедление волны вблизи этих частот. Вблизи частот $\omega_{7}$ и $\omega_{8}$ групповая скорость не только становится больше световой, но и имеет противоположные знаки. Согласно (22) такое поведение $v_{g}$ и $v_{p h}$ объясняется либо сильной нормальной дисперсией, при которой групповая скорость может оказаться намного меньше $c$, либо аномальной дисперсией $\left(d N_{\text {eff }} / d \omega<0\right)$, при которой $v_{g}>c$. Более того, достаточно сильная аномальная дисперсия $\left(N_{\text {eff }}-\left|\omega d N_{\text {eff }} / d \omega\right|<0\right)$ приводит к отрицательному значению $v_{g}$, что принято считать указанием на формирование в рассматриваемой структуре обратной волны, у которой направление потока энергии противоположно направлению фазовой скорости. В слабо диспергирующей среде групповая скорость совпадает со скоростью переноса энергии $v_{e}=S / w$,

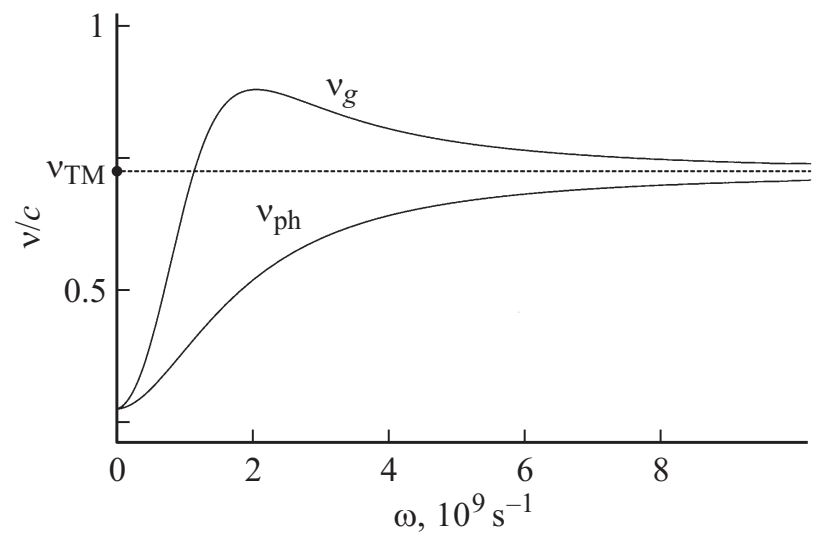

Рис. 6. Частотная зависимость фазовой и групповой скоростей для ТМ волны в структуре при $H=100 \mathrm{Oe}$.

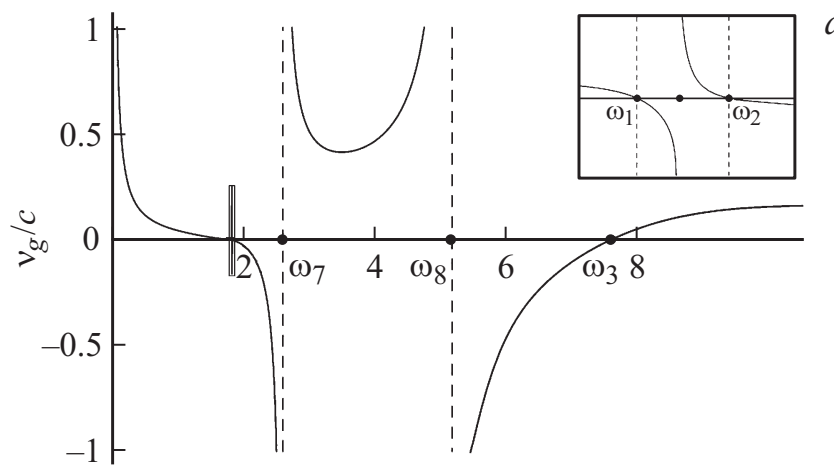

$a$

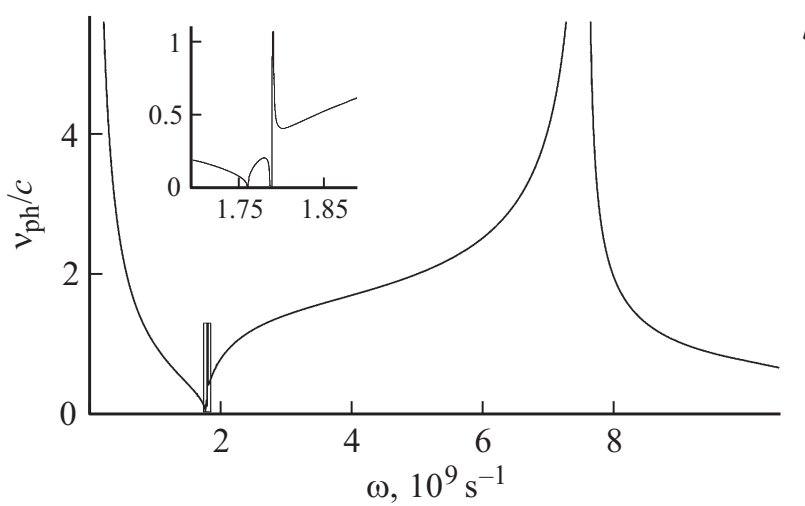

$b$

Рис. 7. Частотная зависимость групповой $(a)$ и фазовой $(b)$ скоростей для ТЕ и волны в структуре при $H=100$ Ое.

где $S$ и $w$ - модуль плотности потока и плотность энергии волны $[25,26]$. В области сильной нормальной и аномальной дисперсий групповая скорость уже не определяет скорость переноса волной „физической субстанции“, а является лишь математическим понятием, определяющим скорость переноса фазовых возмущений в волне [26-28].

\section{Заключение}

В настоящей работе для плоской границы раздела феррита и метаматериала, состоящего из помещенной в немагнитную диэлектрическую матрицу решетки параллельных металлических магнитных микропроводков, определены частотные области существования объемных и поверхностных волн. Проведенный анализ показал, что в такой структуре возможны только объемные волны ТМ типа, тогда как волны ТЕ типа могут быть и объемными, и поверхностными. Волны ТМ типа не управляемы внешним магнитным полем, тогда как ТЕ волны магнитоуправляемы. В частности, с помощью поперечного магнитного поля можно управлять степенью локализации волнового поля вблизи границы раздела сред, групповой и фазовой скоростями распространяющихся волн, сдвигать частотную область существования ПВ в структуре. Объемная ТМ волна имеет только положительную групповую скорость во всем спектральном интервале, тогда как и объемная, и поверхностная ТЕ 
волны могут иметь как положительную, так и отрицательную групповые скорости. И прямые, и обратные волны могут испытывать существенное замедление.

Работа выполнена при финансовой поддержке Министерства образования и науки РФ в рамках проектов № 3.175.2014K, № 14.Z50.31.0015.

\section{Список литературы}

[1] Shelby R.A., Smith D.R., Schultz S. // Science. 2001. Vol. 292. P. 77.

[2] Виноградов А.П. Электродинамика композитных материалов. М.: УРСС Эдиториал, 2001.

[3] Sihvola A. // Metamaterials. 2007. Vol. 1. № 1. P. 2.

[4] Гуляев Ю.В., Лагарьков А.Н., Никитов С.А. // Вестник РАН. 2008. Т. 78. Вып. 5. С. 438.

[5] Иванов А.В., Шалыгин А.Н., Ведяев А.В., Иванов В.А. // Письма в ЖЭТФ. 2007. Т. 85. Вып. 11. С. 694.

[6] Вендик И.Б., Вендик О.Г. // ЖТФ. 2013. Т. 83. Вып. 1. С. 3.

[7] Sarychev A.K., Shalaev V.M. // Phys. Report. 2000. Vol. 335. P. 275.

[8] Caloz C., Itoh T. Electromagnetic metamaterial. NY.: J. Wiley and Sons, 2006.

[9] Filatov L.D., Eliseeva S.V., Sementsov D.I. // Appl. Surf. Sci. 2015. Vol. 351. P. 48.

[10] Башарин А.А., Меньших Н.Л. // Письма в ЖЭТФ. 2011. Т. 93. Вып. 12. С. 770.

[11] Maradudin A.A. Surface waves. Festkoperprobleme. 1981.Vol. 21. P. 25.

[12] Поверхностные поляритоны / Под ред. В.М. Аграновича, Д.Л. Миллса. М.: Наука, 1985.

[13] Nanophotonics with Surface Plasmons / Ed. by V.M. Shalaev, S. Kawata. Amsterdam: Elsevier, 2007.

[14] Zayats A.V., Smolyaninov I.I., Maradudin A.A. // Phys. Rep. 2005. Vol. 408. P. 131.

[15] Жирнов С.В., Семенщов Д.И. // ФТТ. 2007. Т. 49. № 5. C. 773.

[16] Семенов А.А., Карманенко С.Ф., Мелков А.А. и др. // ЖТФ. 2001. T. 71. № 10. C. 13.

[17] Беспятых Ю.И., Бугаев А.С., Дикитейн И.Е. // ФТТ. 2001. T. 43. № 11. C. 2043.

[18] Санников Д.Г., Семенщов Д.И. // ФТТ. 2013. Т. 55. Вып. 11. C. 2209.

[19] Pendry J.B., Holden A.J., Stewart W.J. et al. // Phys. Rev. Lett. 1996. Vol. 76. P. 4773.

[20] Reynet O., Adenot A.L., Deprot S. et al. // Phys. Rev. B. 2002. Vol. 66. P. 0994412.

[21] Makhnovskiy D.P., Panina L.V., Garcia C. et al. // Phys. Rev. B. 2006. Vol. 74. P. 064205.

[22] Молоканов В.В., Умнов П.П., Куракова Н.В. и др. // Перспективные материалы. 2006. Т. 2. С. 5.

[23] Ахиезер А.И., Барьяхтар В.Г., Каганов М.И. // УФН. 1960. Т. 71. Вып. 4. С. 333.

[24] Гуревич А.Г., Мелков Г.А. Магнитные колебания и волны. М.: Наука, 1994.

[25] Ахиезер А.И., Ахиезер И.А. Электромагнетизм и электромагнитные волны. М.: Высшая школа, 1985.

[26] Шевченко В.В. // УФН. 2007. Т. 177. № 4. С. 301.

[27] Давидович М.В. // УФН. 2009. Т. 179. № 4. С. 443.

[28] Давидович М.В. // ЖТФ. 2010. Т. 80. Вып. 5. С. 40. 